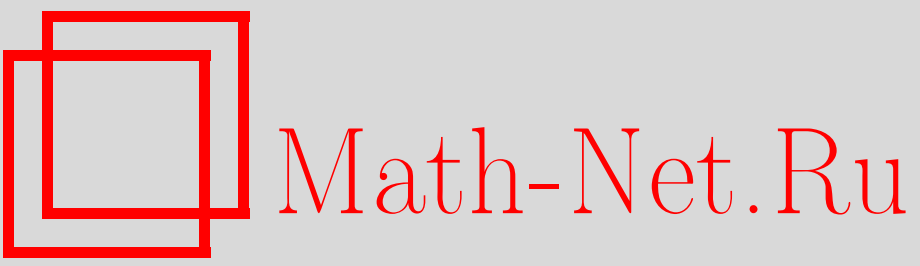

Д. Н. Ахиезер, Э. Б. Винберг, В. В. Горбацевич, В. Г. Дурнев, Р. Зуланке, Л. С. Казарин, Д. А. Лейтес, В. В. Серганова, В. М. Тихомиров, Аркадий Львович Онищик (к 70-летию со дня рождения), УМН, 2003, том 58, выпуск 6, 193200

DOI: https://doi.org/10.4213/rm695

Использование Общероссийского математического портала Math-Net.Ru подразумевает, что вы прочитали и согласны с пользовательским соглашением

http://www.mathnet.ru/rus/agreement

Параметры загрузки:

IP : 54.209 .52 .79

26 апреля 2023 г., $17: 00: 22$ 


\section{АРКАДИЙ ЛЬВОВИЧ ОНИЩИК}

(к семидесятилетию со дня рождения)

В ноябре 2003 г. исполнилось 70 лет профессору Ярославского государственного университета Аркадию Львовичу Онишику.

А. Л. Онищик родился в Москве 14 ноября 1933 г. В 1951 г. он поступил на механико-математический факултет МГУ. Он был одним из лидеров своего курса, активным участником факультетской жизни, руководителем школьных кружков, организатором математических олимпиад. Его научным руководителем был Е. Б. Дынкин, автор классических работ по теории групп Ли. Свои первые научные результаты А. Л. Онищик получил еще в студенческие годы. Некоторые из них вошли в болшшую совместную с Е.Б. Дынкиным статью о глобальном строении компактных групп Ли, опубликованную в "Успехах математических наук" в 1955 г.

В 1956 г. А. Л. Онищик окончил механико-математи-

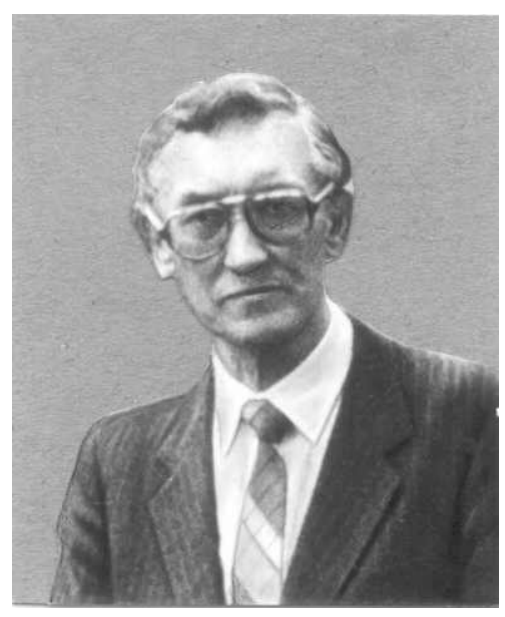
ческий факултет МГУ и поступил в аспирантуру кафедры высшей алгебры. После окончания аспирантуры он был оставлен на кафедре высшей алгебры, которой в те годы заведовал А.Г. Курош.

В 1960 г. А. Л. Онищик защитил кандидатскую диссертацию "О транзитивных группах Ли преобразований", а в 1970 г. - докторскую диссертацию "Компактные однородные пространства и разложения групп Ли”. В 1975 г. он перешел на работу в Ярославский университет, где получил должность профессора.

Одни из первых работ А. Л. Онищика посвящены изучению когомологий пространств петель на топологических пространствах методом спектральных последователшностей. Он изучил важный частный случай, в котором алгебра когомологий пространства петель $\Omega(X)$ может быть определена по алгебре когомологий самого пространства $X$. Именно, предположим, что алгебра $H^{*}(X)$ является свободной антикоммутативной алгеброй конечного типа, и пусть $y_{i}, i=$ $1,2, \ldots,-$ ее свободные образующие. Тогда, как утверждает доказанная им теорема, алгебра $H^{*}(\Omega(X))$ имеет свободную систему образующих $x_{i}$ степеней $\operatorname{deg}\left(x_{i}\right)=\operatorname{deg}\left(y_{i}\right)-1$. Более точно, $x_{i}$ образуют базис пространства трансгрессивных элементов в когомологической спектральной последовательности расслоения пространства путей над $X$, причем при трансгрессии $x_{i}$ соответствует $y_{i}$. Этому резултату предшествовала совместная работа с $\Phi$.И. Карпелевичем, в которой предполагалось, что все $y_{i}$ имеют нечетные степени, т.е. что $H^{*}(X)$ - внешняя алгебра.

В качестве приложения А. Л. Онищик вычислил алгебру $H^{*}(\Omega(M))$, где $M=G / U-$ односвязное однородное пространство полупростой компактной группы Ли $G$. В частности, когда алгебра $H^{*}(M)$ есть тензорное произведение алгебр с одной образующей, он доказал, что алгебра $H^{*}(\Omega(M))$ вьчисляется прямо по $H^{*}(M)$. Отсюда вытекает, что если алгебра $H^{*}(M)$ 
задана, то по одной из двух алгебр $H^{*}(G), H^{*}(U)$ можно определить другую. Эти результаты, опубликованные в 1958 г., положили начало многолетним исследованиям А. Л. Онищика, некоторые итоги которых были подведены в его книге "Топология транзитивных групп преобразований" (1995). Мы вернемся чуть ниже к топологии однородных пространств, а сейчас приведем один фундаменталшный резултат, важный одновременно для комплексного анализа и теории алгебраических групп преобразований, опубликованный в его заметке в Докладах АН СССР в 1960 г.

K этому времени, благодаря проникновению методов теории пучков в теорию функций многих комплексных переменных, стала понятна роль, которую играют в комплексном анализе многообразия Штейна. Первоначально введенные К. Штейном как некоторые обобщения областей голоморфности, эти многообразия составляют, как выяснилось, естественный класс, для которого верны теоремы А и В, доказанные в семинаре А. Картана 1951-1952 гг. Ввиду важности самого понятия, особую роль приобретает задача об описании многообразий Штейна, однородных относительно транзитивной группы Ли голоморфных преобразований. Эта задача в полном объеме не решена и до сих пор даже для комплексных групп Ли преобразований.

В заметке А. Л. Онищика речь идет о многообразиях Штейна, однородных относителњно редуктивных комплексных алгебраических групп. Пусть $M=G / H$ - комплексное однородное пространство такой группы $G$. Результат А. Л. Онишика состоит в следующем: (а) многообразие $M$ является многообразием Штейна тогда и только тогда, когда подгруппа $H$ алгебраична и редуктивна; (б) при выполнении этого условия $M$ обладает структурой аффиннного комплексного алгебраического многообразия. Примерно в то же время эта теорема была независимо доказана Й. Мацусимой. По ходу дела А. Л. Онищик получил следующий важный результат. Пусть $K-$ компактная группа линейных преобразований вещественного векторного пространства $V$. Тогда комплексификация $G$ группы $K$ транзитивно действует на комплексификации орбиты любой точки $v \in V$, причем стационарная подгруппа точки $v$ в группе $G$ совпадает с комплексификацией ее стационарной подгруппы в группе $K$. Этот результат впоследствии передоказывался А. Борелем и Хариш-Чандрой и другими авторами.

Возвращаясь к топологии транзитивных групп преобразований, рассмотрим вначале задачу об отыскании всех разложений вида $G=G^{\prime} \cdot G^{\prime \prime}$, где $G$ - связная компактная группа Ли, $G^{\prime}, G^{\prime \prime}$ - ее связные компактные подгруппы. Эта задача равносильна задаче отыскания связных замкнутых подгрупп группы $G$, транзитивных на однородных пространствах этой группы. Она была полностью решена А. Л. Онищиком в 1962 г. Имеют место следуюшие разложения, часть которых была известна и ранее:

$$
\begin{gathered}
S U_{2 n}=S p_{n} \cdot S U_{2 n-1}(n \geqslant 2) ; S O_{7}=G_{2} \cdot S O_{6} ; S O_{7}=G_{2} \cdot S O_{5} \\
S O_{2 n}=S O_{2 n-1} \cdot S U_{n}(n \geqslant 4) ; S O_{4 n}=S O_{4 n-1} \cdot S p_{n}(n \geqslant 2) ; \\
S O_{16}=S O_{15} \cdot \operatorname{Spin}_{9} ; S O_{8}=S O_{7} \cdot \operatorname{Spin}_{7} .
\end{gathered}
$$

Окончательньй ответ состоит в том, что любое нетривиальное разложение, с точностью до локалњного изоморфизма и перестановки сомножителей, получается из одного из приведенных выше нормальным расширением группы $G^{\prime \prime}$. Примечательно, что доказательство этого алгебраического результата основано на исследовании топологических свойств разложений. Его чисто алгебраического доказательства до сих пор никем не найдено.

Теорема о разложениях компактных групп Ли имеет многочисленные применения. В частности, с ее помощью А. Л. Онищик нашел группы автоморфизмов фолаговых многообразий простых комплексных групп Ли. Он также применил ее к вычислению группы изометрий риманова однородного пространства простой компактной группы Ли.

В задаче о разложениях задана группа Ли, но не фиксировано ее однородное пространство. Можно поступить наоборот и спросить, какие группы Ли действуют транзитивно на заданном однородном пространстве $M=G / H$. В случае, когда $G$ и $H$ - связные компактные группы Ли, эта задача далеко продвинута, причем наиболее полные резултаты здесь принадлежат А. Л. Онищику. Ее решение требует введения инвариантов однородных пространств, которые эффективно вычисляются по заданной паре $(G, H)$, но на самом деле зависят только от $M$. А. Л. Онишиком был введен целочисленный инвариант, названный им рангом однородного пространства. Обозначим через $P_{G}$ пространство примитивных вещественных классов когомологий группы $G$. Тогда 
при отображении $i^{*}: H^{*}(G, \mathbb{R}) \rightarrow H^{*}(H, \mathbb{R})$, индуцированном вложением $i: H \hookrightarrow G$, имеем $i^{*}\left(P_{G}\right) \subset P_{H}$. По определению, ранг $r(M)$ однородного пространства $M=G / H$ равен размерности ядра отображения $\left.i^{*}\right|_{P_{G}}$. Отметим, что $r(G)$ есть ранг группы $G$ в обычном смысле и, кроме того, $r(M)>0$, если $M$ имеет положительную размерность. А. Л. Онишик получил формулы

$$
r(M)=\sum_{k=0}^{\infty} \operatorname{rk} \pi_{2 k+1}(M), \quad h(M):=r(G)-r(H)=\sum_{k=0}^{\infty}(-1)^{k} \operatorname{rk} \pi_{k+1}(M),
$$

из которых видно, что $h(M) \leqslant r(M)$ и что оба инварианта зависят толко от $M$.

А. Л. Онишик нашел все односвязные компактные однородные пространства рангов 1 и 2 . Полученный им список многообразий ранга 1 содержит все сферы, комплексные и кватернионные проективные пространства, октавную проективную плоскость и ряд других многообразий. Для каждого из них он описал все транзитивные действия связных компактных групп Ли. Результаты Д. Монтгомери, Г. Самельсона и А. Бореля о транзитивных группах на сферах включаются в эту теорию как частный случай. Другим ее следствием является тот факт, что гомотопическая эквивалентность двух односвязных компактных однородных пространств ранга 1 равносильна их диффеоморфности (для пространств ранга 2 это уже не так).

Предположим теперь, что для некоторого односвязного компактного однородного пространства $M$ известна классификация связных компактных транзитивных групп Ли преобразований, и рассмотрим вопрос о перенесении ее на некомпактные группы. В такой общности эта задача была впервые поставлена и во многом решена А. Л. Онищиком. Прежде всего, им была доказана следующая теорема (вещественньй аналог теоремы Ж. Титса): если $G$ - связная группа Ли и $H \subset G$ - такая замкнутая подгруппа, что многообразие $M=G / H$ компактно, то нормализатор $N\left(H^{\circ}\right)$ связной компоненты $H^{\circ}$ группы $H$ содержит максимальную связную треугольную подгруппу группы $G$. Из этой теоремы следует, что коммутант радикала $R$ группы $G$ коммутативен. Более того, если многообразие $M$ односвязно и $r(M)=1$, то и сам радикал коммутативен. При этом возможны два случая: (i) $h(M)=1$ и $R$ может иметь сколь угодно большую размерность (например, для нечетномерной сферы); (ii) $h(M)=0$ и $R=\{e\}$, т.е. $G$ полупроста (например, для четномерной сферы). Используя эти результаты, А. Л. Онищик нашел все связные полупростые группы Ли, транзитивно действующие на односвязных компактных однородных пространствах ранга 1, и выяснил, какие из них могут быть расширены до неполупростых групп преобразований. Приведем найденный им список некомпактных простых групп Ли, транзитивных на сферах $S^{n}, n \geqslant 2$. С точностью до локального изоморфизма эти группы таковы:

$$
\begin{gathered}
S O_{1, n+1}^{\circ}, S U_{1, m}(n=2 m-1), S p_{1, m}(n=4 m-1), F I I(n=15), S L_{n+1}(\mathbb{R}), \\
S L_{m}(\mathbb{C})(n=2 m-1), S p_{2 m}(\mathbb{R})(n=2 m-1), S p_{2 m}(\mathbb{C})(n=4 m-1), \\
S L_{m}(\mathbb{H})(n=4 m-1) \text { и универсальная накрывающая группы } S L_{3}(\mathbb{R})(n=3) .
\end{gathered}
$$

Описание всех транзитивных групп на односвязных компактных однородных пространствах ранга больше 1 известно пока лишь в отдельных случаях. Например, А. Л. Онишик перечислил связнье транзитивные группы Ли преобразований вещественных, комплексных и кватернионных многообразий Грассмана и Штифеля. Эти результаты стали основой многочисленных работ других авторов по геометрии, топологии и алгебре.

А. Л. Онищик несколько раз возвращался к задаче о разложениях группы Ли в произведение двух подгрупп, решенной им для компактных групп. В частности, им были описаны все разложения связных редуктивных групп Ли в произведение двух связных редуктивных подгрупп. Оказалось, что эта задача равносильна описанию локальных разложений. Иначе говоря, если $G$ - редук тивная группа, $F$ и $H$ - ее редуктивные подгруппы и группа $F$ имеет в $G / H$ открытую орбиту, то эта орбита совпадает с $G / H$. Этот результат, впоследствии обобщенный другими авторами, имеет принципиальное значение для теории алгебраических групп преобразований. 
А. Л. Онишик был одним из первых, кто стал применять методы неабелевой теории когомологий к конкретным геометрическим задачам. В частности, им получено обобщение теоремы де Рама о существовании и когомологичности замкнутых дифференциальных форм с заданными периодами (для 1-форм), в котором роль форм играют связности без кривизны в заданном расслоенном пространстве, а роль периодов - их группы голономий.

А. Л. Онишику принадлежат важные теоремы конечности в теории инвариантов компактных групп Ли преобразований. Именно, пусть $G$ - компактная группа Ли, действующая на компактном многообразии $M$, и $E$ - векторное $G$-расслоение над $M$ (все объекты - класса $C^{\infty}$ ). Обозначим через $C^{\infty}(M)_{G}$ и $C^{\infty}(M)^{G}$ алгебры гладких сферических и, соответственно, инвариантных функций на $M$. А. Л. Онищик доказал, что модуль гладких инвариантных сечений расслоения $E$ над алгеброй $C^{\infty}(M)^{G}$ имеет конечный тип. Он также доказал, что алгебра $C^{\infty}(M)_{G}$ допускает конечную систему образующих над $C^{\infty}(M)^{G}$.

Ряд работ А. Л. Онищика относительно недавнего времени посвящен теории супермногообразий. Им, в частности, доказана теорема о жесткости некоторых суперграссманианов, предложена новая конструкция нерасшепимых супермногообразий и найдены условия, при которых аналитическое действие группы Ли поднимается на нерасщепимое супермногообразие с его ретракта. В совместной работе с О.В. Платоновой он классифицировал однородные и четно однородные супермногообразия, редукция которых есть проективное пространство.

Педагогическая деятелность А. Л. Онищика началась более 40 лет тому назад на механико-математическом факултете Московского университета. В то время совместно с Э. Б. Винбергом им был организован семинар по группам Ли, ставший преемником семинара Е. Б. Дынкина. Все последующие годы, в частности, и после перехода в Ярославский университет, А. Л. Онищик является одним из руководителей этого семинара. Записки семинара 1967/68 гг. легли в основу книги Э.Б. Винберга и А. Л. Онищика "Семинар по группам Ли и алгебраическим группам" (1988), которая стала незаменимой для аспирантов и специалистов в этой области.

А. Л. Онишик является создателем лаборатории теоретико-групповых методов в Ярославском университете. С первых дней работы в Ярославле он регулярно редактирует и издает сборники научных трудов "Геометрические методы в задачах анализа и алгебры" и "Вопросы теории групп и гомологической алгебры", которые приобрели широкую известность благодаря высокому уровню публикаций. Эти сборники получили международное признание: многие статьи из них были переведены на английский язьк. А. Л. Онищик является также членом редколлегий журналов "Annals of Global Analysis and Geometry" и "Transformation Groups". Как редактор он отличается высокой требовательностью к математическим текстам наряду с чутким и внимателшным отношением к их авторам.

Высокий уровень математики в Советском Союзе был бы недостижим без своевременного и качественного перевода мировой математической литературы на русский язьк. А. Л. Онищик относился к переводу книг как к культрной миссии, а не как к второстепенной временной работе. Читатели книг Чжэнь Шэн-шэня "Комплексные многообразия", С. Хелгасона "Диффференциальная геометрия и симметрические пространства" и других переведенных им книг и статей, несомненно, оценили по достоинству мастерство переводчика. Несколько лет с болњшой пользой для читателей А. Л. Онишик работал библиотекарем Московского математического общества.

Аркадию Львовичу свойственны профессионализм, высокая култура, широта интересов в сочетании с доброжелательностью, отзывчивостью и замечательным чувством юмора. Эти качества всегда привлекали к нему молодежь. За годы работы в Московском и Ярославском университетах он воспитал несколько поколений учеников, многие из которых продолжают активно работать в области математики и ее приложений или преподают математику. Они сохраняют чувство благодарности своему учителю, который не только учил их математике, но своим личным примером, скромно и без риторики, учил уважать человеческую личность.

Мы желаем Аркадию Львовичу доброго здоровья, новых интересных математических результатов и долгих лет жизни, столь же содержательной и плодотворной, как и та ее часть, что осталась позади.

Д. Н. Ахиезер, Э. Б. Винберг, В. В. Горбачевич, В. Г. Дурнев, Р. Зуланке, Л. С. Казарин, Д. Лейтес, В. Серганова, В. М. Тихомиров 


\section{СПИСОК ПУБЛИКАЦИЙ А. Л. ОНИЩИКА}

[1] Об ориентируемости аналитических однородных многообразий // УМН. 1953. Т. 8. C. $121-130$

[2] Компактные группы Ли в целом // УМН. 1955. Т. 10. С. 3-74 (совм. с Е. Б. Дынкиным)

[3] Алгебра гомологий пространства путей // Докл. АН СССР. 1956. Т. 106. С. 967-969 (совм. с $\Phi$. И. Карпелевичем)

[4] Пространства путей и расслоенные пространства // Докл. АН СССР. 1956. Т. 110. C. $932-935$

[5] О когомологиях пространств путей // Матем. сб. 1958. Т. 44. С. 3-52

[6] О транзитивных группах преобразований компактных однородных пространств // Докл. AH CCCP. 1959. T. 124 . C. $520-523$

[7] О включениях между транзитивными компактными группами преобразований // Докл. AH CCCP. 1959. T. 129. C. 261-264

[8] Комплексные оболочки компактных однородных пространств // Докл. АН СССР. 1960. T. 130 . C. $726-729$

[9] О транзитивных группах Ли преобразований // Дисс. ... канд. физ.-матем. наук. М.: МГУ, 1960

[10] О компактных группах Ли, транзитивных на некоторых многообразиях / / Докл. АН СССР. 1960. T. 135 . C. $531-534$

[11] О кручении особых групп Ли // Матем. сб. 1960. Т. 51. С. 273-276

[12] О классификации расслоенных пространств // Докл. АН СССР. 1961. Т. 141. С. 803-806

[13] Отношения включения между транзитивными компактными группами преобразований // Труды ММО. 1962. Т. 11. С. 199-242

[14] Полупростые разложения полупростых алгебр Ли // Докл. АН СССР. 1963. Т. 149. C. $1033-1036$

[15] О транзитивных компактных группах преобразований / Матем. сб. 1963. Т. 60. С. 447-485

[16] Связности без кривизны и теорема де Рама // Докл. АН СССР. 1964. Т. 159. С. 988-991

[17] О деформациях расслоенных пространств. // Докл. АН СССР. 1965. Т. 161. С. 45-47

[18] О деформациях голоморфных расслоенных пространств / Современные проблемы теории аналитических функций. М.: Наука, 1966. С. 236-239

[19] О группах Ли, транзитивных на компактных многообразиях // Матем. сб. 1966. Т. 71. C. $483-494$

[20] О группах Ли, транзитивных на компактных многообразиях. II // Матем. сб. 1967. Т. 74. C. $398-416$

[21] Некоторые понятия и применения теории неабелевых когомологий // Труды ММО. 1967. T. 17. C. $45-88$

[22] О группах Ли, транзитивных на компактных многообразиях. III // Матем. сб. 1968. Т. 75. C. $255-263$

[23] Об однородных векторных расслоениях. // УМН. 1969. Т. 24. № 3. С. 231-232

[24] Семинар по алгебраическим группам и группам Ли, 1967/68 г. М.: МГУ, 1969 (совм. с Э.Б. Винбергом)

[25] Разложения редуктивных групп Ли // Матем. сб. 1969. Т. 80. С. 553-599

[26] Группы Ли, транзитивные на многообразиях Грассмана и Штифеля // Матем. сб. 1970. T. 83 . C. $407-428$

[27] Компактные однородные пространства и разложения групп Ли // Дисс. . . докт. физ.матем. наук. М.: МГУ, 1970

[28] О вполне интегрируемых уравнениях на однородных пространствах // Матем. заметки. 1971. T. 9. C. $365-373$

[29] Мероморфные функции и расслоения на комплексные прямые на компактных комплексных многообразиях // Труды VIII летней матем. школы. Киев, 1971. С. 78-101

[30] О некоторых топологических инвариантах однородных пространств // Матем. заметки. 1972. T. 12 . C. $761-768$ 
[31] Пространства Штейна // Итоги науки и техники. Алгебра, топология, геометрия. Т. 11. М.: ВИНИТИ, 1974. С. 125-151

[32] О полупрямых суммах алгебр Ли // Матем. заметки. 1975. Т. 18. С. $31-40$ (совм. с Ю. Б. Хакимджановым)

[33] Об инвариантах и почти-инвариантах компактных групп преобразований // Труды ММО. 1976. T. 35. C. $235-264$

[34] О расширениях транзитивных групп преобразований // Изв. вузов. Матем. 1977. № 3. С. 53-65; Исправления: 1981. № 7. С. 88

[35] О транзитивных действиях на борелевских многообразиях // Вопр. теории групп и гомолог. алгебры. Ярославл: ЯрГУ, 1977. С. 143-155

[36] Algebra und Geometrie. Eine Einführung. Berlin: DVW, 1977, 1986 (with R. Sulanke)

[37] Псевдовыпуклость в теории комплексных пространств / / Итоги науки и техники. Алгебра, топология, геометрия. Т. 15. М.: ВИНИТИ, 1977. С. 93-171

[38] Элементы линейной алгебры и теории групп. Ярославл: ЯрГУ, 1978

[39] Введение в теорию групп и алгебр Ли. Ярославл: ЯрГУ, 1979

[40] Замечание об инвариантах групп, порожденных отражениями // Вопр. теории групп и гомолог. алгебры. Ярославль: ЯрГУ, 1979. С. 138-141

[41] Классификация компактных групп Ли // Добавление к кн.: Дж. Адамс. Лекции по группам Ли. М.: Наука, 1979. С. 132-144

[42] Линейная алгебра для биологов. Ярославль: ЯрГУ, 1980 (совм. с А. Н. Рудаковым)

[43] О вполне геодезических подмногообразиях симметрических пространств // Геометрические методы в задачах алгебры и анализа. Ярославль: ЯрГУ, 1980. С. 64-85

[44] Об одном классе подгрупп простых алгебраических групп // Вопр. теории групп и гомолог. алгебры. Ярославль: ЯрГУ, 1981. С. 93-103

[45] Parabolic factorizations of semisimple algebraic groups // Math. Nachr. 1981. V. 104. P. $315-329$

[46] О включениях между транзитивными алгебраическими группами // Изв. вузов. Матем. 1982. № 2. C. $28-35$

[47] О транзитивных полупростых группах Ли // Вопр. теории групп и гомолог. алгебры. Ярославл: ЯрГУ, 1983. С. 128-129

[48] О топологии некоторых комплексных однородных пространств // Многомерный комплексный анализ. Красноярск: СО АН СССР, 1985. С. 109-121

[49] Методы теории пучков и пространства Штейна // Итоги науки и техники. Соврем. пробл. матем. Фундам. направления. Т. 10. М.: ВИНИТИ, 1986. С. 5-73

[50] Транзитивные неприводимые супералгебры Ли векторных полей // Деп. в ВИНИТИ 12.06.86, № 4329-В. Ярославль: ЯрГУ, 1986

[51] Приводимые максимальные подалгебры некоторых классических супералгебр Ли // Вопросы теории групп и гомолог. алгебры. Ярославль: ЯрГУ, 1987. С. 161-163 (совм. с О.А. Жаровым, В. А. Золотаревым)

[52] Algebra und Geometrie 2. Moduln und Algebren. Berlin: DVW, 1988 (with R. Sulanke)

[53] Семинар по группам Ли и алгебраическим группам. М.: Наука, 1988 (совм. с Э. Б. Винбергом)

[54] Группы Ли и алгебры Ли - 1: Основы теории групп Ли. Группы Ли преобразований // Итоги науки и техники. Соврем. пробл. матем. Фундам. направления. Т. 20. М.: ВИНИТИ, 1988 (совм. с Э.Б. Винбергом, В. В. Горбацевичем)

[55] Супералгебры Ли векторных полей на расщепимых флаговых супермногообразиях // Докл. АН СССР. 1988. Т. 300. № 2. С. 284-287 (совм. с А. А. Серовым)

[56] On the centre of a transitive semisimple Lie group // Ann. Global Anal. Geom. 1988. V. 6. № 3. P. $265-272$

[57] Действия супералгебр Ли картановского типа на некоторых супермногообразиях // Вопросы теории групп и гомолог. алгебры. Ярославл: ЯрГУ, 1989. С. 42-49

[58] Подалгебры Картана в супералгебрах Ли // Вопр. теории групп и гомолог. алгебры. Ярославль: ЯрГУ, 1989. С. 99-104 (совм. с В. А. Бунегиной, Т. Н. Наумовой) 
[59] Штейновы однородные пространства редуктивных групп // Добавление к кн.: Г. Грауэрт, Р. Реммерт. Теория пространств Штейна. М.: Наука, 1989. С. 328-331

[60] Группы Ли и алгебры Ли - 3 // Итоги науки и техники. Соврем. пробл. матем. Фундам. направления. Т. 41. М.: ВИНИТИ, 1990 (совм. с Э. Б. Винбергом, В. В. Горбацевичем)

[61] Lie Groups and Algebraic Groups. Berlin: Springer-Verlag, 1990 (with E. B. Vinberg)

[62] Lie superalgebras of vector fields // Amer. Math. Soc. Transl. Ser. 2. 1991. V. 148. P. 87-97

[63] Неприводимые компоненты внешней алгебры над пространством матриц // Вопр. теории групп и гомолог. алгебры. Ярославль: ЯрГУ, 1992. С. 136-139

[64] Об одном семействе однородных супермногообразий // Вопр. теории групп и гомолог. алгебры. Ярославль: ЯрГУ, 1992. С. 153-158 (совм. с В. А. Бунегиной)

[65] The group of isometries of a compact Riemannian homogeneous space // Differential Geometry and Its Applications. (Eger, Hungary, 1989). Amsterdam: North Holland, 1992. P. 597-616. (Colloq. Math. Soc. János Bolyai. V. 56.)

[66] Holomorphic vector fields on super-Grassmannians / Lie Groups, Their Discrete Subgroups, and Invariant Theory. Providence, RI: Amer. Math. Soc., 1992 (with A. A. Serov)

[67] On the rigidity of super-Grassmannians // Ann. Global Anal. Geom. 1993. V. 11. P. 361-372

[68] Homogeneous supermanifolds associated with the complex projective line // Preprint № 33. Oslo: Inst. Math. Univ. Oslo, 1993 (with V. A. Bunegina)

[69] Lie Groups and Lie Algebras, I: Foundations of Lie Theory. Li Transformations Groups. Berlin: Springer-Verlag, 1993 (with V. V. Gorbatsevich, E. B. Vinberg)

[70] Lie Groups and Lie Algebras, III: Structure of Lie Groups and Lie Algebras. Berlin: SpringerVerlag, 1994 (with Э. Б. Винберг, В. В. Горбацевич)

[71] Topology of Transitive Transformation Groups. Leipzig: J. A. Barth Verlag, 1994

[72] Flag supermanifolds, their automorphisms and deformations // The Sophus Lie Memorial Conference (Oslo, 1992). Proceedings. Oslo: Scand. Univ. Press, 1994. P. 289-302

[73] Евгений Борисович Дынкин (к 70-летию со дня рождения) // УМН. 1994. Т. 49. С. 183-188 (совм. с Н. Д. Введенской, Р. Л. Добрушиным, В. А. Успенским)

[74] Two families of flag supermanifolds // Differential Geom. Appl. 1994. V. 4. № 4. P. 329-360 (with V.A. Bunegina)

[75] A spectral sequence for the tangent sheaf cohomology of a supermanifold // SFB 288, Preprint № 148. Berlin: Technisce Universität, 1994

[76] Семинар по группам Ли и алгебраическим группам. М.: УРСС, 1995 (совм. с Э.Б. Винбергом)

[77] About derivations and vector-valued differential forms // Bericht № 181. Bohum: Inst. Math. Ruhr-Universität, 1995

[78] О когомологиях касательного пучка комплексного супермногообразия // Актуальные проблемы естественных и гуманитарных наук. Математика, информатика. Ярославл: ЯрГУ, 1995. С. 44-46

[79] Топология транзитивных групп преобразований. М.: Физматлит, 1995

[80] On the work of E. B. Dynkin in the theory of Lie groups // Amer. Math. Soc. Transl. Ser. 2. 1995. V. 169. P. 1-13 (with F. I. Karpelevich, E. B. Vinberg)

[81] Vector fields and deformations of isotropic super-Grassmannians of maximal type // Amer. Math. Soc. Transl. Ser. 2. 1995. V. 169. P. 75-90 (with A. A. Serov)

[82] О комплексных однородных супермногообразиях // Математика в Ярославском университете. Сб. обзорных статей. Ярославл: ЯрГУ, 1996. С. 133-153

[83] On isotropic super-Grassmannians of maximal type associated with an odd bilinear form // Preprint № 340. Vienna: E. Schrödinger International Institute for Mathematical Physics, 1996 (with A. A. Serov)

[84] A construction of non-split supermanifolds // Preprint № 361. Vienna: E. Schrödinger International Institute for Mathematical Physics, 1996

[85] Homogeneous supermanifolds associated with the complex projective line // J. Math. Sci. 1996. V. 82. № 4. P. 3503-3527 (with V. A. Bunegina)

[86] Non-split supermanifolds associated with the cotangent bundle // Prépubl. Université de Poitiers, Départ. de Math., № 109. Poitiers, 1997 
[87] Foundations of Lie Theory and Lie Transformation Groups. 2nd printing. Berlin: SpringerVerlag, 1997 (with V. V. Gorbatsevich, E. B. Vinberg)

[88] About derivations and vector-valued differential forms // J. Math. Sci. (New York). 1998. V. 90. № 4. P. 2274-2286

[89] A spectral sequence for the tangent sheaf cohomology of a supermanifold // Lie Groups and Lie Algebras. Dordrecht: Kluwer, 1998. P. 199-215. (Math. Appl. V. 433.)

[90] Однородные супермногообразия, связанные с комплексным проективньм пространством. I // Матем. сб. 1998. Т. 189. №2 2. С. 111-136 (совм. с О. В. Платоновой)

[91] Однородные супермногообразия, связанные с комплексным проективньп пространством. II // Матем. сб. 1998. Т. 189. № 3. С. 103-124 (совм. с О.В.Платоновой)

[92] A moduli problem related to complex supermanifolds // Algebra and Operator Theory. Proc. colloquium (Tashkent, 1997). Dordrecht: Kluwer, 1998. P. 13-24

[93] A construction of non-split supermanifolds // Ann. Global Anal. Geom. 1998. V. 16. № 4. P. 309-333

[94] On non-abelian cochain complexes // Вопросы теории групп и гомолог. алгебры. Ярославль, 1998. С. $171-197$

[95] Non-abelian cohomology and supermanifolds // SFB 288, Preprint 360. Berlin: Technische Universität, 1998

[96] On the classification of complex analytic supermanifolds // Lobachevskii J. Math. 1999. V. 4. P. $47-70$

[97] On supermanifolds associated with the cotangent bundle // Lie Groups, Geometric Structures and Differential Equations - One Hundred Years after Sophus Lie (Kyoto, 1999). Kyoto: RIMS, 2000. P. 45-53. (Søurikaisekikenkyøusho Køokyøuroku. V. 1150.)

[98] Lifting of holomorphic actions on complex supermanifolds // Preprint 966. Vienna: E. Schrödinger International Institute for Mathematical Physics, 2000

[99] Треугольные когомологии компактных кэлеровых многообразий / / Матем. сб. 2001. Т. 192. № 2. С. 27-56 (совм. с А. Ю. Брудным)

[100] Проблемы классифокиации комплексных супермногообразий // Математика в Ярославском университете. Сб. обзорных статей. K 25-летию математического факултета. Ярославль: ЯрГУ, 2001. С. 7-33

[101] Lifting of holomorphic actions on complex supermanifolds // Lie Groups, Geometric Structures and Differential Equations - One Hundred Years after Sophus Lie (Kyoto, 1999). Tokyo: Math. Soc. Japan, 2002. P. 317-335. (Adv. Stud. Pure Math. V. 37.) 